\title{
Influence of Frequency on the Resolution of Magnetostrictive Bio-Inspired Whisker Sensors
}

\author{
Ran Zhao $\mathbb{D}^{1,2}$ Bo-wen Wang, ${ }^{2}$ Quan-guo Lu, ${ }^{1}$ Jian-wu Yan, ${ }^{1}$ and Xiao-cui Yuan ${ }^{1}$ \\ ${ }^{1}$ Jiangxi Province Key Laboratory of Precision Drive \& Control, Nanchang Institute of Technology, Nanchang, China \\ ${ }^{2}$ State Key Laboratory of Reliability and Intelligence of Electrical Equipment, Hebei University of Technology, Tianjin, China \\ Correspondence should be addressed to Ran Zhao; zhaoran@nit.edu.cn
}

Received 18 December 2017; Revised 9 June 2018; Accepted 28 June 2018; Published 7 August 2018

Academic Editor: Maria Luz Rodriguez-Mendez

Copyright ( 2018 Ran Zhao et al. This is an open access article distributed under the Creative Commons Attribution License, which permits unrestricted use, distribution, and reproduction in any medium, provided the original work is properly cited.

\begin{abstract}
Magnetostrictive biomimetic whiskers have been used as tactile and flow sensors. Compared to other types of whiskers, such whiskers have the advantage of being able to perform static and dynamic measurements. For dynamic measurement, the whisker's resolution changes with varying vibration frequency; however, the mechanism for this influence has not been studied yet. Thus, the aim of this study is to investigate the resolution-frequency correlation. First, the structure and operation principle of the whisker were analyzed. Then, the Euler-Bernoulli beam theory was employed to establish the sensing model of the magnetostrictive whisker. Finally, the mapping relationship between sensor resolution and frequency was obtained. The eigenfrequency analysis was implemented by FEM to obtain the frequency response of the whisker. A vibration experimental system was built for dynamic testing. The experimental results were in good agreement with the theoretical calculations. Furthermore, it was noted that the resolution was positively correlated with frequency, and the maximum resolution was attained at the natural frequency (two peak values appeared at the first-order and second-order eigenfrequencies). Our research reveals the manner in which a whisker sensor's resolution is affected by the vibration frequency. The theoretical model can be used to predict the resolution of magnetostrictive whisker sensors.
\end{abstract}

\section{Introduction}

In nature, several animals use their whiskers to collect ambient information, including obstacles, distance, and force and vibration, to overcome any limitations of vision and hearing. For example, seals use their whiskers to perceive hydrodynamics, and rats rely on whiskers to recognize obstacles. Many researchers have studied the operation mechanism of animal whiskers and developed artificial whisker systems. As a near-range sensor, the biomimetic whisker can be a useful supplement for traditional sensing technologies.

In the last two decades, research on bio-inspired whiskers has progressed greatly. The principle, materials, measurement methods, and applications of biomimetic whiskers have been studied extensively. Among them, Lucianna et al and Kan et al. investigated the mechanism and elastic modulus of a rat whisker sensory system $[1,2]$. Bovet et al. established the simulation models of rigid and flexible whiskers [3]. Kaneko and Tsuji first used a flexible beam coupling with a torque sensor to detect the hole shape in machining processes; the whisker had a high sensitivity of $5 \mu \mathrm{m}[4,5]$. Ju and Ling developed a piezoelectric whisker and applied it to tactile and geometry measurements $[6,7]$. Another kind of tactile sensor, a PVDF beam-based whisker was reported, and its frequency response was studied by Tiwana et al. [8]. Pearson et al. and N'Guyen et al. applied the whisker sensory system in a robotic rat for obstacle identification $[9,10]$. However, all the abovementioned whiskers are based on elastic or piezoelectric materials, and their application range is limited.

It is known that magnetostrictive devices exhibit excellent static and dynamic performances [11-13]. This characteristic makes them potentially applicable in the sensing field. To this end, Raghunath and coworkers developed a magnetostrictive whisker [14]. On the basis of magnetoelastic effect mechanism, a magnetostrictive whisker can measure both stress and vibration. As a novel whisker-type tactile sensor, it can sense static and dynamic information, 
and it demonstrates satisfactory environmental adaptability. In these studies, the whisker's magnetoelastic bending model and design optimization problems (the location of the bias magnetic field and the relation between sensor sensitivity and beam thickness $[15,16])$ were investigated; however, the dynamic performance (such as dynamic sensitivity and resolution) has not been studied yet. In [17], we discussed the influence of frequency on the sensitivity of the magnetostrictive whisker, but the mapping relation between the vibration frequency and whisker response is still unclear.

To realize the quantitative description of a whisker's resolution, we fabricated, analyzed, and modeled a galfenol(FeGa alloy-) based magnetostrictive whisker sensor. The frequency response of the whisker was obtained by using a finite element software. A dynamic experimental system was established to test the dynamic performance of the whisker. The details are presented in the following sections.

\section{Design}

2.1. Structure and Fabrication. Figure 1 shows a typical magnetostrictive whisker consisting of four parts: a magnetostrictive cantilever beam, a bias magnet, and a magnetic sensor. Several magnetostrictive materials, such as galfenol and alfenol (Fe-Al alloy) $[15,16]$, can be used as raw materials of the whisker beam. The beam was clamped at the base and magnetized by the bias magnet. The change in magnetic flux was measured by a magnetic sensor. Either a Hall sensor or a GMR sensor can be used as the magnetic sensors. Here, we selected a Hall sensor, the WSH138 (manufactured by Winson Lt. Co.) to measure the change in magnetic flux [18].

In the design process, a high magnetostrictive coefficient and flexibility are the two mainly considered factors. Thus, we selected a galfenol thin plate to fabricate the beam. The magnetostrictive coefficient of galfenol is higher than that of alfenol (their saturation magnetostrictions are 250 and $120 \mathrm{ppm}$, respectively, reported in [19]). The rolling process ensures that this material demonstrates excellent elasticity. The geometric and physical parameters of the magnetostrictive whisker are listed in Table 1.

2.2. Operation Principle. Figure 2 illustrates the operation principle of the proposed whisker. The bias magnet provides a magnetic field to orient the magnetic domains along the $y$ axis direction. The linkage flux is detected by a Hall sensor fixed next to it (Figure 2(a)). Raghunath et al. observed the change in magnetic domain in a bending magnetostrictive beam using a Kerr microscope [20]. It was found that the domain rotation depends on the inner stress distribution. As shown in Figure 2(b), the magnetic domains on the right side of the natural centerline have the maximum deflection angle. Thus, the linkage flux increases. In contrast, when stress is applied to the beam rightwards, this value decreases (Figure 2(c)). In summary, when stress is applied from different directions, the Hall sensor outputs an alternating voltage.

The measurement method of the proposed whisker allows the sensor to measure both static and dynamic stresses. Furthermore, the microsecond response speed of

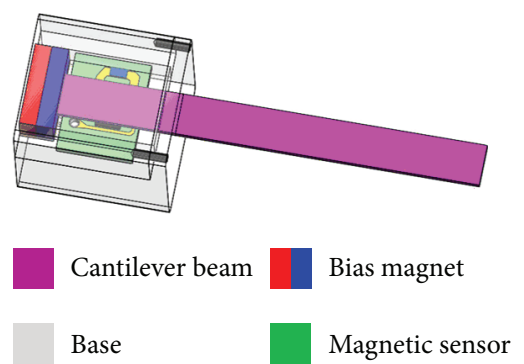

FIGURE 1: 3D model of the magnetostrictive whisker.

galfenol and Hall sensor can help the whisker realize realtime monitoring tasks.

2.3. Definition of Resolution. In our design, the whisker transforms the displacement signal to a voltage signal. In addition, its displacement resolution is dependent on the minimum conversion voltage of the measurement circuit. This means that the displacement resolution of the whisker system is limited by the resolution of the Hall sensor. Herein, we introduce the beam's displacement-linkage flux transmission coefficient $\alpha$ and the Hall's magnetic resolution $r_{\mathrm{H}}$. The system resolution can be expressed as

$$
r=\alpha \cdot r_{\mathrm{H}},
$$

where $\alpha$ is defined as $d y / d B$, that is, the differential of the displacement to the magnetic flux, and the magnetic resolution of WSH138 is $0.1 \mathrm{mT}$ [18]. From (1), it is known that the system resolution depends on the system sensitivity and the resolution of the Hall sensor.

\section{Analytical Modeling}

3.1. Classic Beam Theory. In this section, a classical beam theory, in the form of the Euler-Bernoulli beam equation, is introduced to model the proposed magnetostrictive whisker. Considering that the designed whisker has a low working frequency, we use a pseudostatic analysis method. For this method, the base vibration excitation (along the direction of the $z$-axis) is equivalent to a dynamic load applied to the free end of the beam [21]. In this way, the static beam equation can be used to model the whisker [22].

According to the Euler-Bernoulli theory, the stress in the $x$-direction of the beam is defined as

$$
\sigma_{x}=-z \frac{M}{I}
$$

where $M$ is the bending moment, and $M=F(L-x)$; $I$ represents the second moment of area. Substituting $M=F(L-x)$ into (2), the stress can be rewritten as

$$
\sigma_{x}(t)=-z \frac{(L-x)}{I} F(t)
$$

where $z$ is the position along the thickness direction, and $F(t)$ represents the equivalent dynamic load, reflecting the effect of the ambient vibration excitation. 
TABle 1: Parameters of the magnetostrictive whisker.

\begin{tabular}{|c|c|c|c|c|}
\hline Material & Name & Symbol & Value & Unit \\
\hline \multirow{2}{*}{ Bias magnet } & Magnetic field strength & $H_{b}$ & 300 & $\mathrm{mT}$ \\
\hline & Dimension & $L \times W \times H$ & $8.5 \times 3.5 \times 2$ & $\mathrm{~mm}^{3}$ \\
\hline \multirow{5}{*}{ Galfenol } & Saturation magnetostriction & $\lambda_{s}$ & 170 & ppm \\
\hline & Young's modulus & $E$ & 60 & $\mathrm{GPa}$ \\
\hline & Poisson’s ratio & $v$ & 0.3 & - \\
\hline & Relative permeability & $\mu_{r}$ & 200 & - \\
\hline & Dimension & $\mathrm{L} \times W \times H$ & $80 \times 4 \times 0.15$ & $\mathrm{~mm}^{3}$ \\
\hline
\end{tabular}

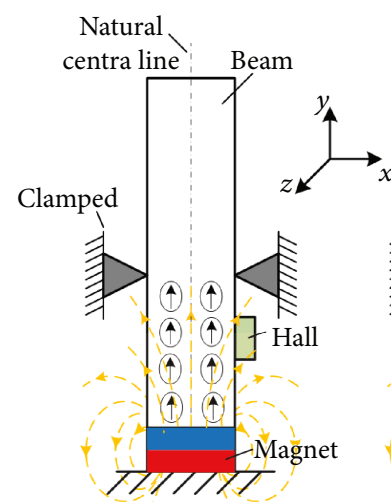

(a)

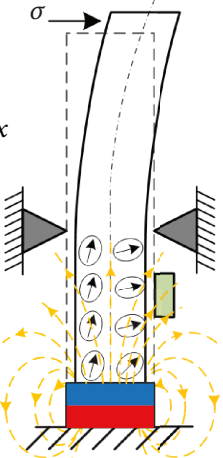

(b)

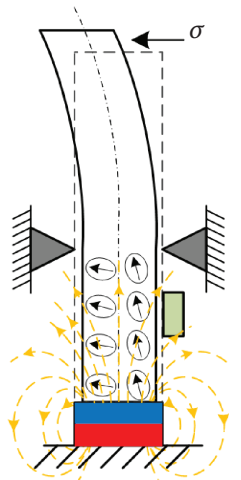

(c)

FIGURE 2: 3D model of the magnetostrictive whisker.

As mentioned in [21], the vibration excitation is described as an input harmonic base motion of

$$
y_{b}(t)=Y_{b} \sin \omega_{b} t, \ddot{y}_{b}(t)=-Y_{b} \omega_{b}^{2} \sin \omega_{b} t .
$$

The tip deflection, $y(t)$, can be obtained by the motion equation of the beam due to a base excitation for acceleration $-Z_{b} \omega_{b}^{2}$ at a frequency of $\omega_{b}$ as

$$
y(t)=Y_{b} a \sin \left(\omega_{b} t-\varphi\right),
$$

where $\omega_{1}$ is the beam's first-order natural frequency; $\varphi=$ $\tan ^{-1}\left(2 \varsigma \omega_{1} \omega_{b} /\left(\omega_{n}{ }^{2}-\omega_{b}{ }^{2}\right)\right)+\tan ^{-1}\left(\omega_{1} / 2 \varsigma \omega_{b}\right), \quad \varsigma$ represents the beam's damping ratio, and $a=\omega_{1}\left[\omega_{1}^{2}+4 \varsigma^{2} \omega_{b}^{2} /\left(\left(\omega_{1}^{2}-\right.\right.\right.$ $\left.\left.\left.\omega_{b}^{2}\right)^{2}+4 \varsigma^{2} \omega_{1}^{2} \omega_{b}^{2}\right)\right]^{1 / 2}$.

The equivalent force can be expressed as

$$
F(t)=\frac{3 E I}{L^{3}}\left(y(t)-y_{b}(t)\right)
$$

By substituting (4), (5), and (6) into (3), we obtain

$$
\sigma_{x}(t)=Y_{b} \frac{3(L-x) \mathrm{z} E}{L^{3}}\left\{\sin \omega_{b} t-a \sin \left(\omega_{b} t-\varphi\right)\right\} .
$$

Most resonant-based energy harvesters demonstrate their highest performance when excited near their resonant frequency. The ith resonance frequency of a uniform beam can be estimated using

$$
\omega_{n}=\beta_{n}^{2} \sqrt{\frac{E I}{m L^{4}}}
$$

where $\beta_{n}$ is the solution of the equation $\cosh \left(\beta_{n} L\right) \cos$ $\left(\beta_{n} L\right)+1=0$.

3.2. Magnetoelastic Sensing Model. The magnetoelastic effect is the basis for the magnetostrictive whisker to realize force sensing. In general, the behavior of magnetostrictive materials is described by the piezomagnetic equation. Using this linear equation, Downey and Flatau derived the expression for the magnetic flux of a bending beam under a bias magnetic field [23]; the formula for which is

$$
B_{\mathrm{i}}(t)=d_{33}^{*} \sigma_{x}(t)-d_{33}^{*} d_{33} E H_{\mathrm{i}}+\mu H_{\mathrm{i}}
$$

where $B_{\mathrm{i}}$ is the internal magnetic flux of the beam. $d_{33}$ and $d^{*}{ }_{33}$ are the piezomagnetic and inverse piezomagnetic coefficients, respectively; they are defined as $d_{33}=$ $\partial \varepsilon / \partial H$ and $d^{*}{ }_{33}=\partial B / \partial H . H_{\mathrm{i}}$ is the internal magnetic field of the beam, and $\mu$ is the permeability of the magnetostrictive material.

Assuming that the bias magnetic and flux are $H_{0}$ and $B_{0}$, $B_{l}$ is the linkage flux in the space; $B_{t}$ is the linkage flux at the 


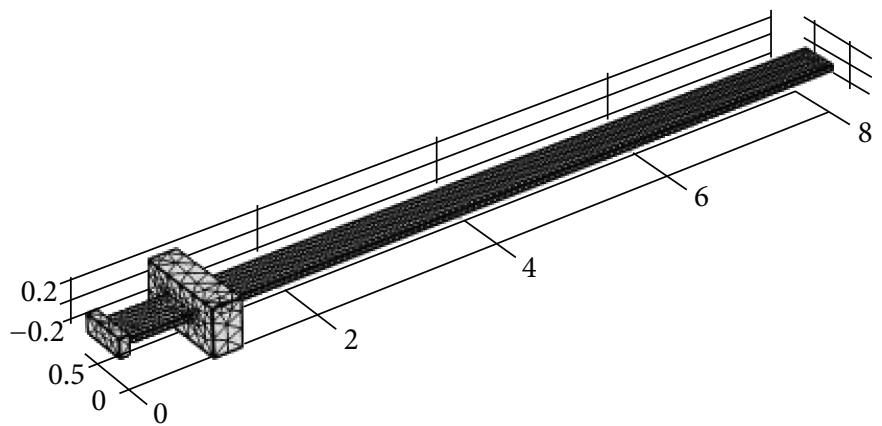

FIgure 3: 3D model and mesh of the magnetostrictive whisker.

measurement point; and $B_{0}=B_{i}+B_{l}$; the linkage flux $B_{t}$ can be expressed as

$$
B_{t}(t)=\frac{1}{2} k_{\mathrm{m}}\left(B_{0}-B_{\mathrm{i}}(t)\right),
$$

where $k_{\mathrm{m}}$ is the linkage magnetic coefficient related to the location of the Hall sensor, relative permeability of galfenol, and the magnetic field distribution. According to (4), (10), and (11), the transform coefficient $\alpha$ can be given by

$$
\begin{aligned}
\alpha(t)= & \frac{d y_{b}(t)}{d B_{t}(t)}=\frac{1}{6} k_{\mathrm{m}} \frac{L^{3}}{(x-L) \mathrm{zE}} \\
& \cdot \frac{1}{\sqrt{(1-a \cos \varphi)^{2}+a^{2} \sin ^{2} \varphi}} \cdot \frac{\cos \omega_{b} t}{\cos \left(\omega_{b} t+\phi\right)},
\end{aligned}
$$

where $\phi=\tan ^{-1}(a \sin \varphi /(1-a \cos \varphi))$, and $x=0$.

Considering that $\alpha(t)$ is a time-varying function, in order to obtain the frequency characteristics of the whisker resolution, we perform a Laplace transform of $\alpha(t)$ and note that $s=j \omega$. Then, we achieve the modular $|\alpha(j \omega)|$; substitute it into (2), and the whisker's displacement resolution can be obtained as follows:

$$
r=|\alpha(j \omega)| \cdot r_{\mathrm{H}}=\frac{1}{6} k_{\mathrm{m}} \frac{L^{2}}{\mathrm{zE}} \cdot \frac{1}{\sqrt{(1-a \cos \varphi)^{2}+a^{2} \sin ^{2} \varphi}} \cdot r_{\mathrm{H}} .
$$

\section{Finite Element Analysis}

For the finite element analysis, a more sophisticated model of the whisker was established in the COMSOL. The eigenfrequency analysis was implemented using structural mechanics, dynamics, and vibration modules. Figure 3 shows the $3 \mathrm{D}$ model and mesh creation of the whisker model. A fine mesh was created with 5501 tetrahedral elements, 3976 boundary triangular elements, 572 mesh points, and 34,302 degrees of freedom. The minimum element quality was set to 0.066 . The model was created using the Lagrange quadratic element type. The computations employed second-order polynomials, which was a good tradeoff between the memory and result accuracy. The use of finer meshes did not have a significant effect on the results. The simulation was performed to calculate the eigenfrequencies for the first six modes.
The COMSOL model also enables the analysis of the shape of the whisker at different modes, as shown in Figure 4. Using the model, the natural frequency of the whisker can be set to any value between 0.5 and $500 \mathrm{~Hz}$ by choosing different materials and physical dimensions.

The theoretical value of the eigenfrequency can be obtained using an analytical model based on (8). The simulated and theoretical values for the six modes are listed and compared in Table 2. The relative error shows that at the first-order natural frequency, theoretical calculation results do not agree well with the simulation result. With the increase in frequency, the results of theoretical models and finite element analysis approach equivalence.

\section{Experiment}

An experimental system was established to test the resolution performance of the proposed magnetostrictive whisker. Figure 5 shows the vibration test system. In this system, the sinusoidal signal was generated by the dSPACE (ds1103) and then amplified by an amplifier (AE7224) to drive the vibration exciter in order to simulate the ambient vibration. The vibration amplitude of the vibration excitation was measured by a laser displacement sensor (Ltc-025). The data from the Hall sensor and laser sensor were acquired using the dSPACE platform. The vibration frequency was set in the range of $0.5-60 \mathrm{~Hz}$. To measure the minimum displacement, for each frequency, the voltage amplitude of the Hall sensor was required to be maintained at $8.3 \mathrm{mV}$.

In the experiment, some parameters required for the theoretical model were also determined. The damping coefficient $\varsigma$ of the whisker system was determined as 0.014 using the Rayleigh model. The linkage magnetic coefficient is $k_{\mathrm{m}}=0.6$. Further, according to the data reported in [24], the inverse piezomagnetic coefficient is $d^{*}{ }_{33}=20 \mathrm{~T} / \mathrm{N}$.

\section{Results and Discussion}

Figure 6 shows the dynamic performance of the magnetostrictive whisker sensor. The voltage amplitude of the Hall sensor was limited to $8.3 \mathrm{mV}$. Then, we obtained the amplitude of the ambient vibration at different frequencies. At $0.5 \mathrm{~Hz}$, which represents the quasi-static condition, the amplitude of the ambient vibration was $1.22 \mathrm{~mm}$. When the frequency became $60 \mathrm{~Hz}$, this value changed to $0.18 \mathrm{~mm}$. It 

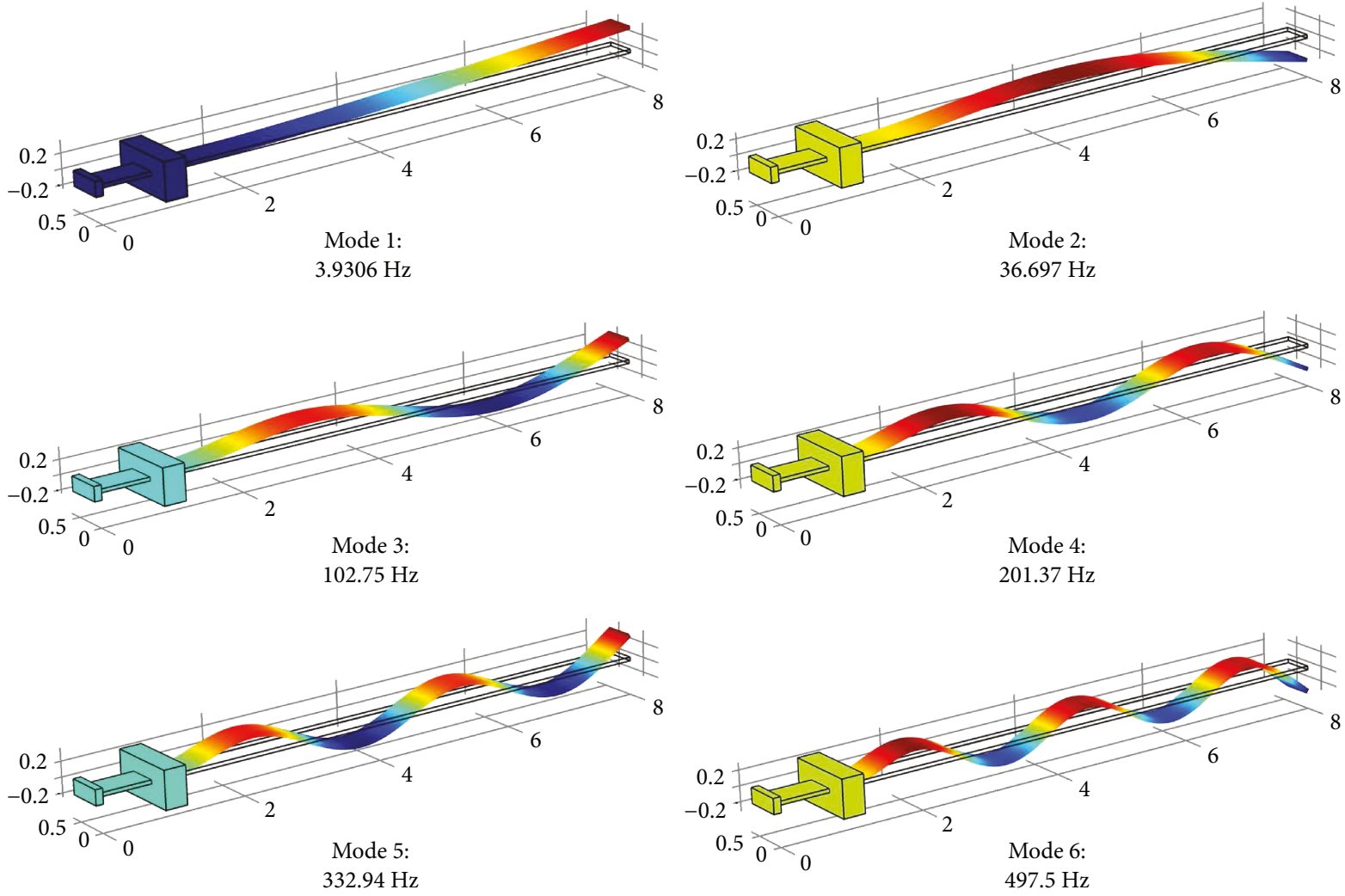

FIGURE 4: Shape of the whisker beam at the first six modes.

TABLE 2: Eigenfrequency calculated using analytical model and finite element software.

\begin{tabular}{lccc}
\hline Mode & Simulated $(\mathrm{Hz})$ & Theoretical $(\mathrm{Hz})$ & Error $(\%)$ \\
\hline 1st & 3.9306 & 4.297 & 9.32 \\
2nd & 36.697 & 35.374 & 3.60 \\
3rd & 102.75 & 99.049 & 3.60 \\
4th & 201.37 & 194.09 & 3.62 \\
5th & 332.94 & 322.29 & 3.19 \\
6th & 497.5 & 483.52 & 2.81 \\
\hline
\end{tabular}

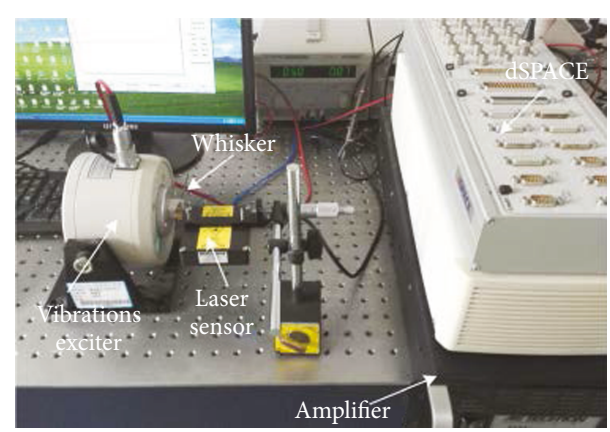

FIGURE 5: Vibration test system for magnetostrictive whisker.

was noted that with increasing frequency, the displacement amplitude sensed by the magnetostrictive whisker decreased gradually. It is thus concluded that the frequency and resolution are positively correlated.
The theoretical value and the experimental results are shown in Figure 7. The figure displays a clear change in the resolution, depending on the vibration frequency. The results of the experiment show the existence of two peaks: $P_{1}$ $(0.18 \mathrm{~mm})$ at $3.5 \mathrm{~Hz}$ and $P_{2}(0.06 \mathrm{~mm})$ near $40 \mathrm{~Hz}$. These two peaks, located near the 1st and 2 nd natural frequencies of the whisker, are the maximum values of the displacement resolution. This means that at natural frequencies, the whisker demonstrates a larger response amplitude than that at other frequencies. The theoretical calculation is in good agreement with the actual value, but only $P_{1}$ occurs in the theoretical curve. This is because our analytical model does not consider additional vibration modes (besides the 1st order natural frequency) for the cantilever beam.

\section{Conclusion}

This study aimed to determine the influence of the vibration frequency on the displacement resolution of the whisker sensor. We established an analytical model based on the EulerBernoulli beam theory to obtain the mapping relation of the sensor resolution and frequency. To analyze the frequency response, we used the finite element method. Further, according to the vibration mode analysis, the first-order to sixth-order natural frequencies were identified. A vibration test system was constructed to determine the dynamic performance of the whisker. Finally, the following conclusions were derived:

(i) The displacement resolution of the whisker sensor is positively correlated. Moreover, the theoretical 


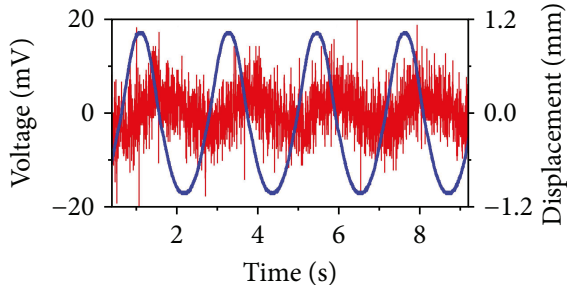

(a)

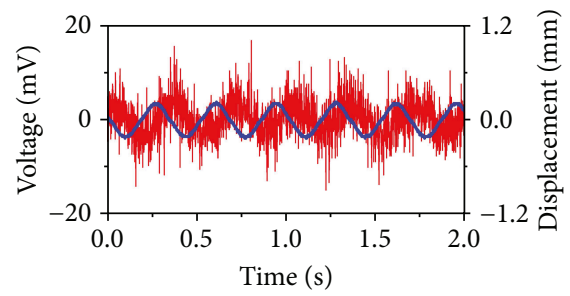

(d)

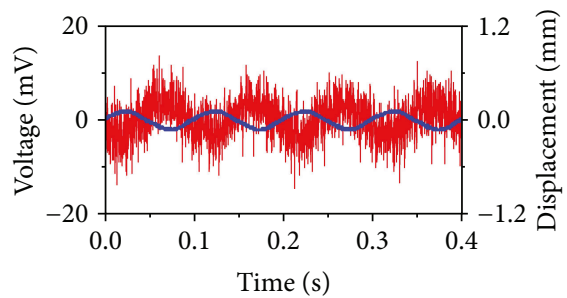

(g)

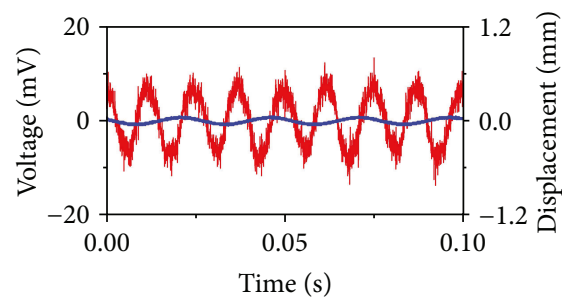

(j)

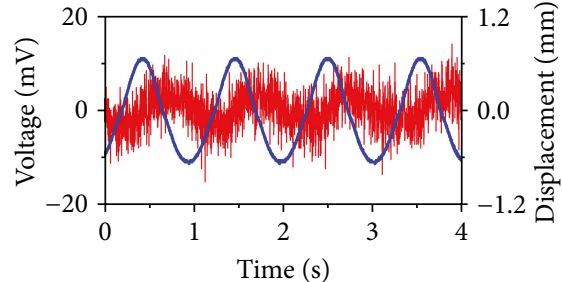

(b)

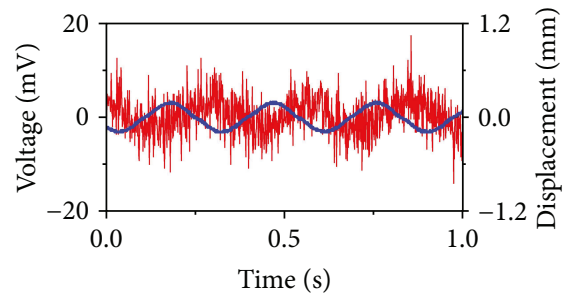

(e)

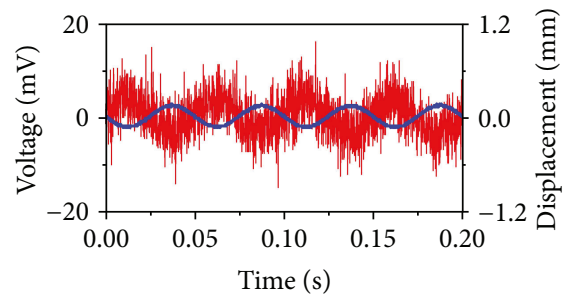

(h)

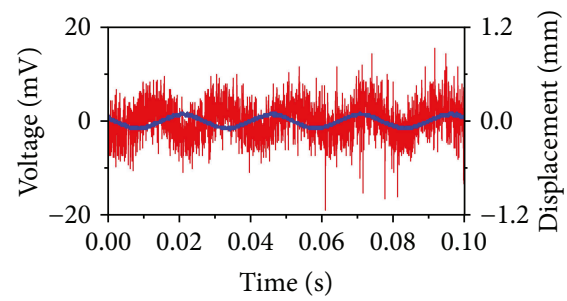

(k)

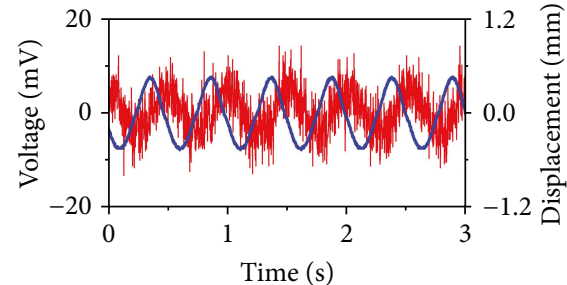

(c)

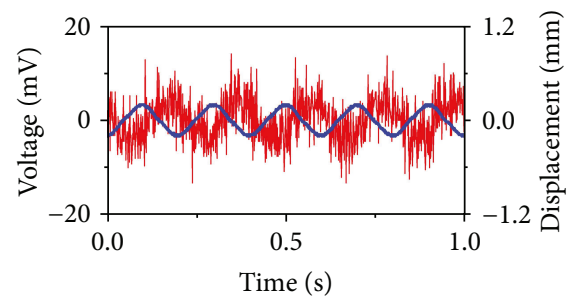

(f)

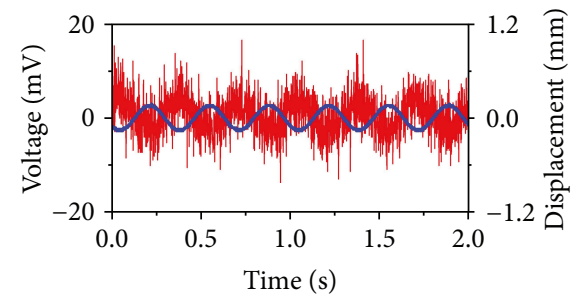

(i)

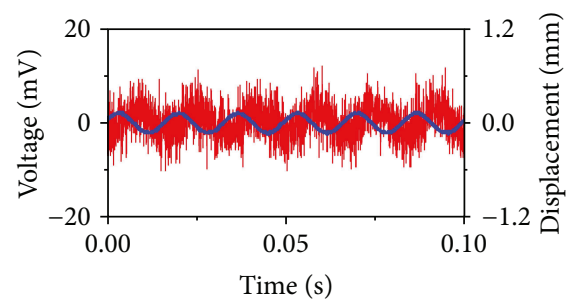

(1)

Figure 6: Sensing voltage and vibration amplitude at various frequencies: (a) 0.5, (b) 1, (c) 2, (d) 3, (e) 3.5, (f) 5, (g) 10, (h) 20, (i) 30, (j) 40, (k) 50, and (l) $60 \mathrm{~Hz}$.

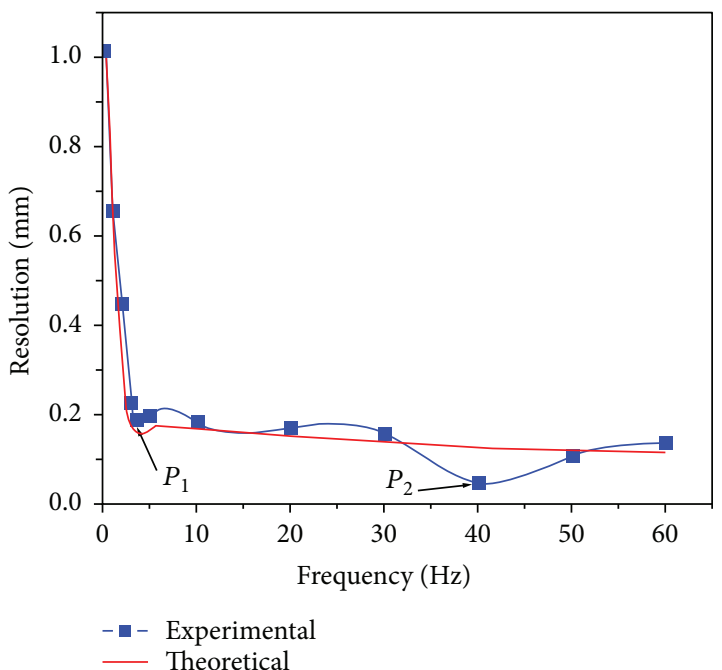

Figure 7: Vibration amplitude resolution dependent on frequency. model presented in this work appropriately conformed to this trend.

(ii) When operating at the resonance frequencies, the resolution attains a local maximum value. For example, in the experiment, two peaks were noted in the vibration amplitude-frequency curve at the 1st and 2nd resonance frequencies of the whisker sensor. That means that the resolution performance of the whisker sensor can be improved by a reasonable frequency design.

(iii) Our research can help understand the dynamic behavior of magnetostrictive whisker sensors and predict its dynamic resolution.

\section{Conflicts of Interest}

The authors declare that they have no conflicts of interest. 


\section{Acknowledgments}

This work was supported by the Science and Technology Project of Jiangxi Province Education Department of China (Grant no. GJJ161105), Natural Science Foundation of Hebei Province (Grant no. E2017202035), the University Science and Technology Projects of Jiangxi Province (Grant nos. KJLD14094 and KJLD14096), and the Open Fund of Jiangxi Province Key Laboratory of Precision Drive \& Control (Grant no. KFKT-201601).

\section{References}

[1] F. A. Lucianna, A. L. Albarracín, S. M. Vrech, F. D. Farfán, and C. J. Felice, "The mathematical whisker: a review of numerical models of the rat's vibrissa biomechanics," Journal of Biomechanics, vol. 49, no. 10, pp. 2007-2014, 2016.

[2] Q. Kan, R. Rajan, J. Fu, G. Kang, and W. Yan, "Elastic modulus of rat whiskers-a key biomaterial in the rat whisker sensory system," Materials Research Bulletin, vol. 48, no. 12, pp. 5026-5032, 2013.

[3] S. Bovet, M. Fend, and R. Pfeifer, "Simulating whisker sensors - on the role of material properties for morphology, behavior and evolution," Journal of the American Veterinary Medical Association, vol. 166, no. 5, pp. 481-486, 2004.

[4] M. Kaneko and T. Tsuji, "A whisker tracing sensor for manufacturing application," IFAC Proceedings Volumes, vol. 33, no. 26, pp. 383-388, 2000.

[5] M. Kaneko and T. Tsuji, "A whisker tracing sensor with $5 \mu \mathrm{m}$ sensitivity," in Proceedings 2000 ICRA. Millennium Conference. IEEE International Conference on Robotics and Automation. Symposia Proceedings (Cat. No.00CH37065), pp. 3907-3912, San Francisco, CA, USA, April 2000.

[6] F. Ju and S. F. Ling, "Bioinspired active whisker sensor for geometry detection of high aspect ratio microholes with simultaneous actuation and sensing capability," Smart Materials and Structures, vol. 24, no. 3, article 035010, 2015.

[7] F. Ju and S. F. Ling, "Bioinspired active whisker sensor for robotic vibrissal tactile sensing," Smart Materials and Structures, vol. 23, no. 12, article 125003, 2014.

[8] M. I. Tiwana, M. I. Tiwana, S. J. Redmond, N. H. Lovell, and J. Iqbal, "Bio-inspired PVDF-based, mouse whisker mimicking, tactile sensor," Applied Sciences, vol. 6, no. 10, pp. 297312, 2016.

[9] M. J. Pearson, A. G. Pipe, C. Melhuish, B. Mitchinson, and T. J. Prescott, "Whiskerbot: a robotic active touch system modeled on the rat whisker sensory system," Adaptive Behavior, vol. 15, no. 3, pp. 223-240, 2007.

[10] S. N'Guyen, P. Pirim, and J. Meyer, “Texture discrimination with artificial whiskers in the robot-rat Psikharpax," in Biomedical Engineering Systems and Technologies. BIOSTEC 2010. Communications in Computer and Information Science, vol 127, A. Fred, J. Filipe, and H. Gamboa, Eds., pp. 74-81, Springer, Berlin, Heidelberg, 2010.

[11] H. Zhang, T. Zhang, and C. Jiang, "Magnetostrictive actuators with large displacement and fast response," Smart Materials and Structures, vol. 21, no. 5, article 055014, 2012.

[12] T. Zhang, C. Jiang, X. Liu, and H. Xu, "Dynamic magnetostrain properties of giant magnetostrictive alloy actuators for damping," Smart Materials and Structures, vol. 14, no. 4, pp. N38-N41, 2005.
[13] H. Zhang, T. Zhang, and C. Jiang, "Design of a uniform bias magnetic field for giant magnetostrictive actuators applying triple-ring magnets," Smart Materials and Structures, vol. 22, no. 11, article 115009, 2013.

[14] G. Raghunath, A. B. Flatau, S. M. Na, and B. Barkley, "Development of a bio-inspired tactile magnetostrictive whisker sensor using alfenol," in ASME Conference on Smart Materials, Adaptive Structures and Intelligent Systems, Newport, RI, USA, September 2014.

[15] P. R. Downey and A. B. Flatau, "Bending of iron-gallium (galfenol) alloys for sensor applications," in Proceeding SPIE 6174, Smart Structures and Materials 2006: Sensors and Smart Structures Technologies for Civil, Mechanical, and Aerospace Systems, San Diego, CA, USA, April 2006.

[16] S. M. Na, M. Rice, G. Raghunath, V. Klimchenko, and A. B. Flatau, "Magnetostrictive alfenol whisker sensor performance and sensitivity to whisker thickness," IEEE Transactions on Magnetics, vol. 50, no. 11, pp. 1-4, 2014.

[17] R. Zhao, Q. Yuan, J. Yan, and Q. Lu, "The static and dynamic sensitivity of magnetostrictive bioinspired whisker sensor," Journal of Nanotechnology, vol. 2018, Article ID 2591080, 6 pages, 2018.

[18] http://www.winson.com.tw/Data\%20Sheet/WSH138.pdf.

[19] N. Srisukhumbowornchai and S. Guruswamy, "Large magnetostriction in directionally solidified FeGa and FeGaAl alloys," Journal of Applied Physics, vol. 90, no. 11, pp. 5680-5688, 2001.

[20] G. Raghunath, M. Marana, S.-M. Na, and A. Flatau, "Kerr microscopy studies of the effects of bending stress on galfenol," Journal of Applied Physics, vol. 115, no. 17, article 17E310, 2014.

[21] J.-H. Yoo and A. B. Flatau, “A bending-mode galfenol electric power harvester," Journal of Intelligent Material Systems and Structures, vol. 23, no. 6, pp. 647-654, 2012.

[22] C. Qinghua, C. Dingfang, L. Quanguo et al., "Sensor performance of cantilevered magnetostrictive beam," International Journal on Smart Sensing and Intelligent Systems, vol. 7, no. 3, pp. 1221-1238, 2014.

[23] P. R. Downey and A. B. Flatau, "Bending behavior of irongallium (galfenol) alloys for sensor applications," in Proceeding SPIE 5764, Smart Structures and Materials 2005: Smart Structures and Integrated Systems, pp. 120-129, San Diego, CA, USA, May 2005.

[24] S. Datta and A. B. Flatau, "Application of iron-gallium alloy as magnetostrictive sensors," in Proceedings SPIE 5764, Smart Structures and Materials 2005: Smart Structures and Integrated Systems, pp. 110-110, San Diego, CA, USA, May 2005. 


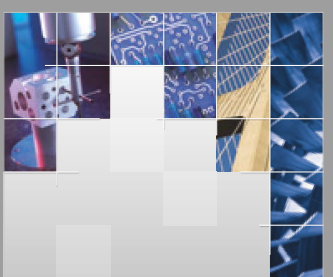

\section{Enfincering}
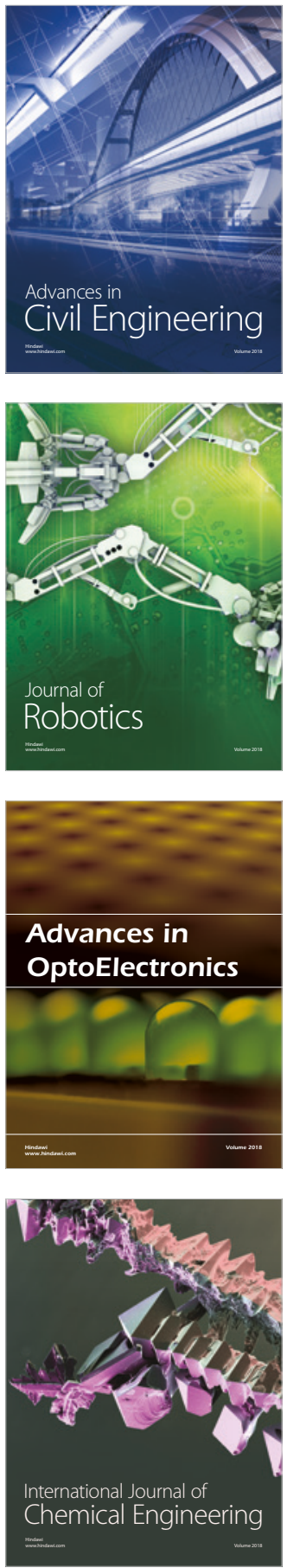

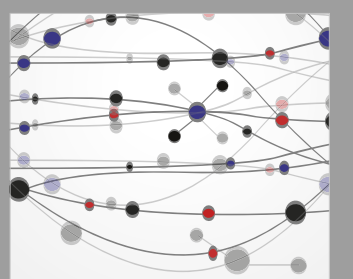

\section{Rotating \\ Machinery}

The Scientific World Journal

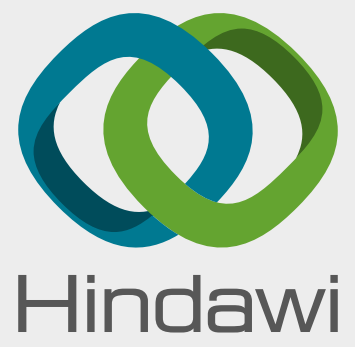

Submit your manuscripts at

www.hindawi.com
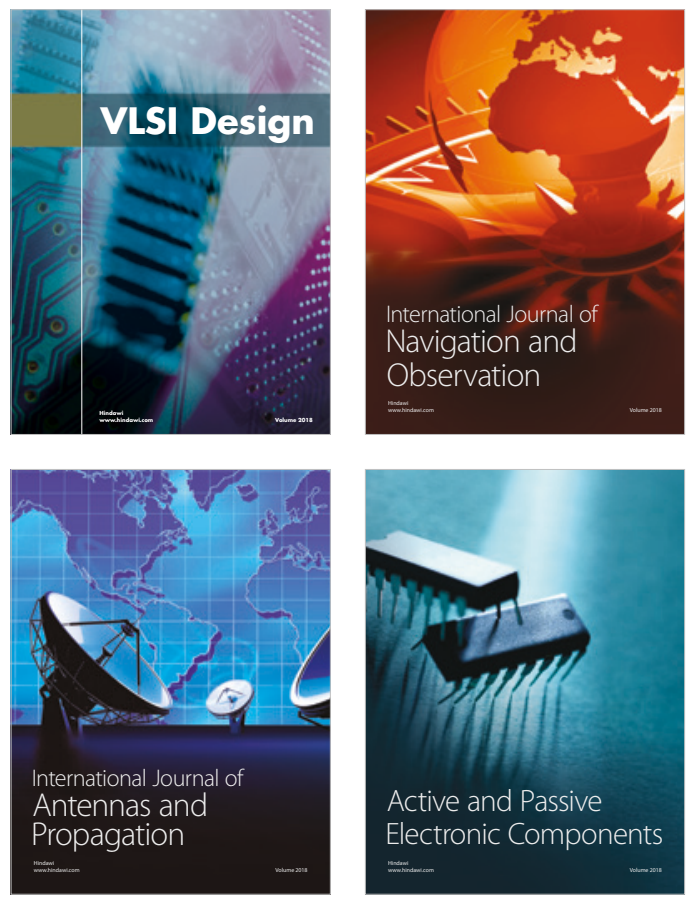
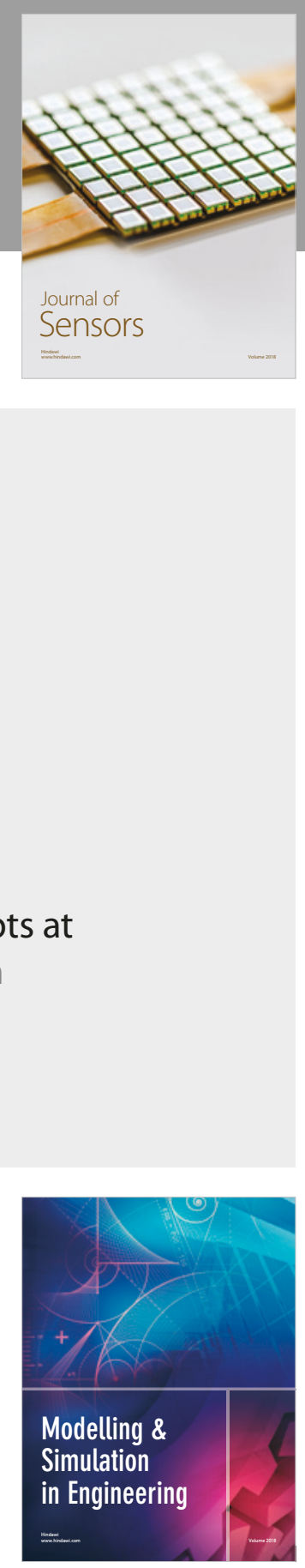

\section{Advances \\ Multimedia}
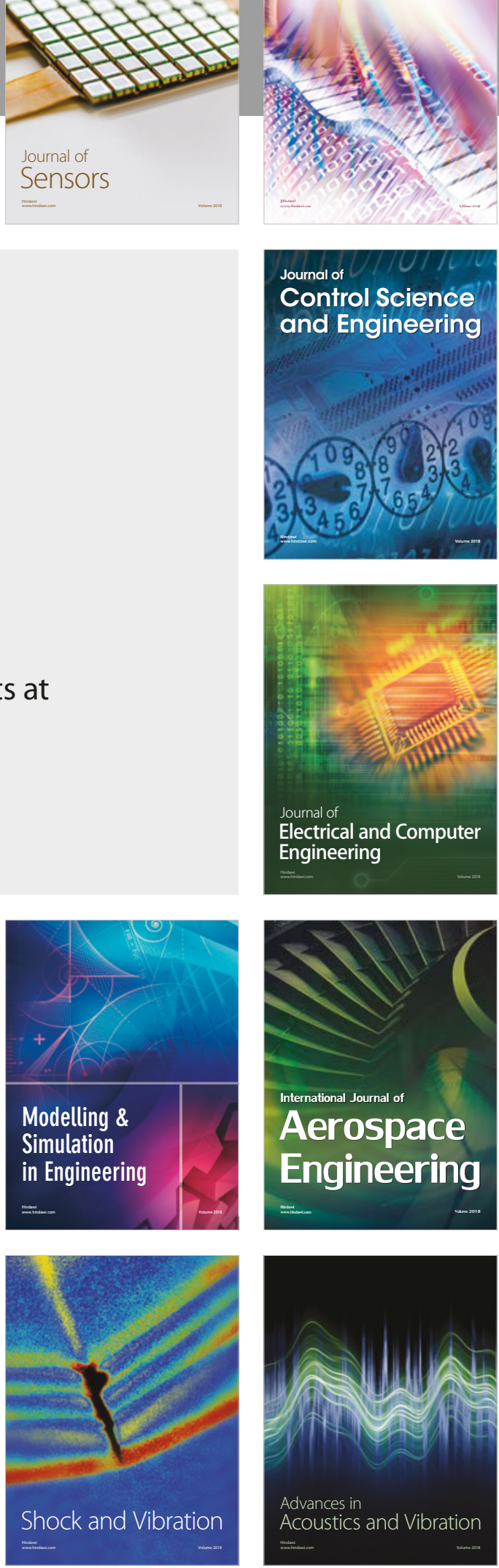